\title{
ON THE GEOMETRY OF THE SPACE OF ORIENTED LINES OF THE HYPERBOLIC SPACE
}

\author{
MARCOS SALVAI* \\ FAMAF - CIEM, Ciudad Universitaria, 5000 Córdoba, Argentina \\ e-mail:salvai@mate.uncor.edu
}

(Received 3 November, 2006; revised 13 March, 2007; accepted 19 March, 2007)

\begin{abstract}
Let $H$ be the $n$-dimensional hyperbolic space of constant sectional curvature -1 and let $G$ be the identity component of the isometry group of $H$. We find all the $G$-invariant pseudo-Riemannian metrics on the space $\mathcal{G}_{n}$ of oriented geodesics of $H$ (modulo orientation preserving reparametrizations). We characterize the null, timeand space-like curves, providing a relationship between the geometries of $\mathcal{G}_{n}$ and $H$. Moreover, we show that $\mathcal{G}_{3}$ is Kähler and find an orthogonal almost complex structure on $\mathcal{G}_{7}$.
\end{abstract}

2000 Mathematics Subject Classification. 53A55, 53C22, 53C35, 53C50, 53D25.

1. Introduction. Let $M$ be a Hadamard manifold (a complete simply connected Riemannian manifold with nonpositive sectional curvature) of dimension $n+1$. An oriented geodesic $c$ of $M$ is a complete connected totally geodesic oriented submanifold of $M$ of dimension one. We may think of $c$ as the equivalence class of unit speed geodesics $\gamma: \mathbb{R} \rightarrow M$ with image $c$ such that $\{\dot{\gamma}(t)\}$ is a positive basis of $T_{\gamma(t)} c$ for all $t$. Let $\mathcal{G}=\mathcal{G}(M)$ denote the space of all oriented geodesics of $M$. The space of geodesics of a manifold all of whose geodesics are periodic with the same length is studied with detail in [1]. The geometry of the space of oriented lines of Euclidean space is studied in $[3,9,10]$.

Let $T^{1} M$ be the unit tangent bundle of $M$ and $\xi$ the spray of $M$, that is, the vector field on $T^{1} M$ defined by $\xi(v)=d /\left.d t\right|_{0} \gamma_{v}^{\prime}(t)$, where $\gamma_{v}$ is the unique geodesic in $M$ with initial velocity $v$. Clearly, $\mathcal{G}$ may be identified with the set of oriented leaves of the foliation of $T^{1} M$ induced by $\xi$. By [7], if $M$ is Hadamard, this foliation is regular in the sense of Palais [8]. Hence, $\mathcal{G}$ admits a unique differentiable structure of dimension $2 n$ such that the natural projection $T^{1} M \rightarrow \mathcal{G}$ is a submersion.

Fix $o \in M$ and let $\operatorname{Exp}: T_{o} M \rightarrow M$ denote the geodesic exponential map. Let $S=\left\{v \in T_{o} M \mid\|v\|=1\right\} \cong S^{n}$. We identify as usual $T_{v} S \cong v^{\perp} \subset T_{o} M$. Hence, $T S \cong$ $\{(v, x) \mid v \in S$ and $\langle v, x\rangle=0\}$. Let $F: T S \rightarrow \mathcal{G}$ be defined by

$$
F(v, x)=[\gamma],
$$

where $\gamma$ is the unique geodesic in $M$ with initial velocity $\tau_{0}^{1} v$ (here $\tau$ denotes parallel transport along the geodesic $t \rightarrow \operatorname{Exp}(t x)$ of $M)$. This is called the minitwistor construction in [5]. Keilhauer proved in [7] that $F$ is a diffeomorphism.

${ }^{*}$ Partially supported by FONCyT, Antorchas, CIEM (CONICET) and SECyT (UNC). 
2. The geometry of $\mathcal{G}$ for the hyperbolic space. Let $H=H^{n+1}$ be the hyperbolic space of constant sectional curvature -1 and dimension $n+1$. Consider on $\mathbb{R}^{n+2}$ the basis $\left\{e_{0}, e_{1}, \ldots, e_{n+1}\right\}$ and the inner product whose associated norm is given by $\|x\|=$ $\langle x, x\rangle=-x_{0}^{2}+x_{1}^{2}+\cdots+x_{n+1}^{2}$. Then $H=\left\{x \in \mathbb{R}^{n+2} \mid\|x\|=-1\right.$ and $\left.x_{0}>0\right\}$ with the induced metric. Let $G$ be the identity component of the isometry group of $H$, that is,

$$
G=O_{o}(1, n+1)=\left\{g \in O(1, n+1) \mid\left(g e_{0}\right)_{0}>0 \text { and } \operatorname{det} g>0\right\} .
$$

In the following we denote $\mathcal{G}_{m}=\mathcal{G}\left(H^{m}\right)$ (or simply $\mathcal{G}$ if no confusion is possible). The group $G$ acts on $\mathcal{G}$ as follows: $\boldsymbol{g}[\gamma]=[g \circ \gamma]$. This action is transitive, since $H$ is two-point homogeneous, and smooth, since $G$ acts smoothly on $T^{1} H$.

Let $\gamma_{o}$ be the geodesic in $H$ with $\gamma_{o}(0)=e_{0}$ and initial velocity $e_{1} \in T_{e_{0}} H$. The isotropy subgroup of $G$ at $c_{o}:=\left[\gamma_{o}\right]$ is

$$
G_{o}=\left\{\operatorname{diag}\left(T_{t}, A\right) \mid t \in \mathbb{R}, A \in S O_{n}\right\} \cong \mathbb{R} \times S O_{n},
$$

where $T_{t}=\left(\begin{array}{cc}\cosh t & \sinh t \\ \sinh t & \cosh t\end{array}\right)$. Therefore we may identify $\mathcal{G}$ with $G / G_{o}$ in the usual way. Let $\mathfrak{g}$ be the Lie algebra of $G$ and let

$$
\mathfrak{g}_{o}=\left\{\operatorname{diag}(t R, A) \mid t \in \mathbb{R}, A \in s o_{n}\right\}
$$

be the Lie algebra of $G_{o}$ (here $\left.R=\left(\begin{array}{ll}0 & 1 \\ 1 & 0\end{array}\right)\right)$. Let $B$ be the bilinear form on $\mathfrak{g}$ defined by $B(X, Y)=\frac{1}{2} \operatorname{tr}(X Y)$, which is well-known to be a multiple of the Killing form of $\mathfrak{g}$, hence nondegenerate. Besides, the canonical projection $\pi: G \rightarrow H, \pi(g)=g\left(e_{0}\right)$, is a pseudo-Riemannian submersion.

Let $\mathfrak{g}=\mathfrak{g}_{0} \oplus \mathfrak{h}$ be the orthogonal decomposition with respect to $B$. Then

$$
T_{c_{o}} \mathcal{G}=\mathfrak{h}:=\left\{x_{h}+y_{v} \mid x, y \in \mathbb{R}^{n}\right\},
$$

where for column vectors $x, y \in \mathbb{R}^{n}$,

$$
x_{h}=\left(\begin{array}{cc}
0_{2} & (x, 0)^{t} \\
(x, 0) & 0_{n}
\end{array}\right) \text { and } y_{v}=\left(\begin{array}{cc}
0_{2} & (0, y)^{t} \\
(0,-y) & 0_{n}
\end{array}\right)
$$

(here the exponent $t$ denotes transpose and $0_{m}$ the $m \times m$ zero matrix). We chose this notation since $x_{h}$ and $y_{v}$ are horizontal and vertical, respectively, tangent vectors in $T_{\left(e_{0}, e_{1}\right)}\left(T^{1} H\right)$ with respect to the canonical projection $T^{1} H \rightarrow H$.

THEOREM 1. For each $n \geq 1$ there exists a G-invariant pseudo-Riemannian metric $g_{1}$ on $\mathcal{G}_{n+1}$ whose associated norm at $c_{o}$ is given by

$$
\left\|x_{h}+y_{v}\right\|_{1}=|x|^{2}-|y|^{2} .
$$

For $n=2$, if one identifies $\mathbb{R}^{2}=\mathbb{C}$ as usual, there exists a $G$-invariant metric $g_{0}$ on $\mathcal{G}_{3}$ whose associated norm at $c_{o}$ is given by

$$
\left\|x_{h}+y_{v}\right\|_{0}=\langle i x, y\rangle .
$$

For $n \neq 2$, any $G$-invariant pseudo-Riemannian metric on $\mathcal{G}_{n+1}$ is homothetic to $g_{1}$. Any $G$-invariant pseudo-Riemannian metric on $\mathcal{G}_{3}$ is of the form $\lambda g_{0}+\mu g_{1}$ for some $\lambda, \mu \in \mathbb{R}$ not simultaneously zero. 
All the metrics are symmetric and have split signature $(n, n)$. In particular, $\mathcal{G}$ does not admit any $G$-invariant Riemannian metric and the geodesics in $\mathcal{G}$ through $c_{o}$ are exactly the curves $s \mapsto \exp _{G}(s X) c_{o}$, for $X \in \mathfrak{h}$.

Proof. One computes easily that $B(X, X)=\|X\|_{1}$ for all $X \in \mathfrak{h}$. Since $B$ is $G$ invariant, $g_{1}$ defines a $G$-invariant metric on $\mathcal{G}$.

Let $Z=\operatorname{diag}\left(R, 0_{n}\right), \mathfrak{m}=\left\{\operatorname{diag}\left(0_{2}, A\right) \mid A \in \operatorname{so} o_{n}\right\}$ and $\mathfrak{g}_{\lambda}=\left\{U \in \mathfrak{g} \mid \operatorname{ad}_{Z} U=\lambda U\right\}$. One verifies that $\mathfrak{g}_{0}=\mathfrak{g}_{o}$ and $\mathfrak{g}_{ \pm 1}=\left\{x_{h} \pm x_{v} \mid x \in \mathbb{R}^{n}\right\}$. Moreover, one has the decompositions

$$
\mathfrak{g}_{0}=\mathbb{R} Z \oplus \mathfrak{m} \quad \text { and } \quad \mathfrak{h}=\mathfrak{g}_{1} \oplus \mathfrak{g}_{-1},
$$

which are preserved by the action of $\mathfrak{m}$. Hence $\mathfrak{h}$ is $\mathfrak{g}_{0}$-invariant.

Since $B$ is nondegenerate and $G_{o}$ is connected, any other pseudo-Riemannian metric $g$ on $\mathcal{G}$ has the form $g(U, V)=B(T U, V)$ for some $T: h \rightarrow h$ commuting with $\operatorname{ad}_{Z}$ and $\operatorname{ad}_{\mathfrak{m}}$. In particular, $T$ preserves $\mathfrak{g}_{ \pm 1}$. We call $T_{ \pm}$the restrictions of $T$ to the corresponding subspaces. Under the identification $\mathfrak{g}_{ \pm 1} \equiv \mathbb{R}^{n}, x_{h} \pm x_{v} \equiv x$, the action of $\mathfrak{m} \equiv s o_{n}$ on $\mathbb{R}^{n}$ is the canonical one. If $T_{ \pm} \in G l\left(\mathfrak{g}_{ \pm 1}\right) \equiv G l(n, \mathbb{R})$ commutes with every $A \in s o_{n}$, then either $T_{ \pm}$is a nonzero multiple of the identity or $n=2$ and $T_{ \pm}=a_{ \pm} I_{2}+b_{ \pm} J$ where $J=\left(\begin{array}{cc}0 & -1 \\ 1 & 0\end{array}\right)$, for some not simultaneously zero constants $a_{ \pm}$ and $b_{ \pm}$. Next we consider the case $n=2$ and show that $a_{+}=a_{-}$and $b_{-}=-b_{+}$. For $x \neq 0$ we denote $x^{ \pm}=x_{h} \pm x_{v}$ and compute

$$
\begin{aligned}
B\left(T\left(x^{+}\right), x^{-}\right) & =B\left(\left(a_{+} x+b_{+} i x\right)^{+}, x^{-}\right) \\
& =a_{+} B\left(x^{+}, x^{-}\right)+b_{+} B\left((i x)^{+}, x^{-}\right) \\
& =2 a_{+}|x|^{2}+0 .
\end{aligned}
$$

Since $T$ must be symmetric with respect to $B$, this expression coincides with $B\left(x^{+}, T\left(x^{-}\right)\right)$, which by similar computations equals $2 a_{-}|x|^{2}$. Hence $a_{+}=a_{-}$. Using again the symmetry of $T$ in the case

$$
B\left(T\left(x^{-}\right),(i x)^{+}\right)=B\left(x^{-}, T(i x)^{+}\right)
$$

one obtains that $b_{-}=-b_{+}$. Finally, since $2\left(x_{h}+y_{v}\right)=(x+y)^{+}+(x-y)^{-}$, one computes that the metric associated with $T$ is homothetic to $g_{1}$ if $b_{+}=0$ and to $g_{o}$ if $a_{+}=0$. The case $n \neq 2$ is simpler since it does not involve $b_{ \pm}$.

Next we show that for any of the metrics above, $\mathcal{G}$ is a symmetric space. Let $G^{\uparrow}=$ $\left\{g \in O(1, n+1) \mid\left(g e_{0}\right)_{0}>0\right\}$ be the isometry group of $H$ and let $C=\operatorname{diag}\left(I_{2},-I_{n}\right) \in$ $G^{\uparrow}$, which induces an involutive diffeomorphism $\widetilde{C}$ of $\mathcal{G}$ by $\widetilde{C}[\gamma]=[C \circ \gamma]$ fixing exactly $c_{o}$. If $n=2, C \in G$, hence $\widetilde{C}$ is clearly an isometry for any $G$-invariant metric on $\mathcal{G}_{3}$. The same happens for $n \neq 2$. Indeed, in this case, up to homotheties, we have seen that the unique metric on $\mathcal{G}_{m}$ with $m \neq 3$ comes from a multiple of the Killing form of $\mathfrak{g}$, which is invariant by the action of $G^{\uparrow}$. The statement regarding geodesics follows from the theory of symmetric spaces, since conjugation by $C$ is an involutive automorphism of $\mathfrak{g}$ whose $(-1)$-eigenspace is $\mathfrak{g}_{0}$ and preserves the given metrics.

REMARKS. a) In contrast with the space of oriented lines of $\mathbb{R}^{n}$, which only for $n=3,7$ admits pseudo-Riemannian metrics invariant by the induced transitive action of a connected closed subgroup of the identity component of the isometry group (see [9]), $\mathcal{G}_{n}$ admits $G$-invariant metrics for all $n$. 
b) The metric $g_{0}$ is the analogue of the metric defined in the Euclidean case in $[11,4]$. We will see below that also in the hyperbolic case it admits a Kähler structure.

c) For any complete simply connected Riemannian manifold $M$ of negative curvature, the space $\mathcal{G}(M)$ of its oriented geodesics has a canonical pseudoRiemannian metric, which is in general only continuous, see [6]. If $M$ is the hyperbolic space, then $g_{1}$ is the canonical metric on $\mathcal{G}$.

d) If $H$ has dimension two, then $\mathcal{G}$ is isometric to the two-dimensional de Sitter sphere.

We recall some well-known facts about the imaginary border of the hyperbolic space and the action of $G$ on it. For a geodesic $\gamma$ in $H, \gamma(\infty)$ is defined to be the unique $z \in S^{n}$ such that $\lim _{t \rightarrow \infty} \gamma(t) / \gamma(t)_{0}=e_{0}+z \in \mathbb{R}^{n+2}$. One defines $\gamma(-\infty)$ analogously. Sometimes we will identify $\mathbb{R}^{n+1}$ with $e_{0}^{\perp}$ and $S^{n}$ with $\left\{e_{0}\right\} \times S^{n}$.

The group $G$ acts on $S^{n}$ by directly (that is, orientation preserving) conformal diffeomorphisms. More precisely, any $g \in G$ induces the directly conformal transformation $\widetilde{g}$ of $S^{n}$, well-defined by $\widetilde{g}(\gamma(\infty))=(g \circ \gamma)(\infty)$, and any directly conformal transformation of $S^{n}$ can be realized in this manner.

Proposition 2. If $S$ is a subgroup of $G$ acting transitively on $\mathcal{G}$, then $S=G$.

Proof. By the main result of [2], it suffices to show that $S$ acts irreducibly on $\mathbb{R}^{n+2}$. Suppose that $S$ leaves the nontrivial subspace $V$ invariant. If $V$ is degenerate, then $V$ contains a null line, say $\mathbb{R}\left(e_{0}+z\right)$, with $z \in \mathbb{R}^{n+1},|z|=1$. Hence $S$ takes the oriented line $[\gamma]$ with $\gamma(\infty)=z$ to another line with the same point at $\infty$. If $V$ is nondegenerate, either $V$ or its complement (also $S$-invariant) intersects $H$. Let us call $H_{1} \varsubsetneqq H$ the intersection, which is a totally geodesic submanifold of $H$. Then $S$ takes any oriented line contained in $H_{1}$ to a line contained in $H_{1}$. If $H_{1}$ is a point $p$, then $S$ takes any line through $p$ to a line through $p$. Therefore the action of $S$ on $\mathcal{G}$ is not transitive.

REMARK. The hyperbolic case contrasts with the Euclidean one. We found in [9] a pseudo-Riemannian metric on the space of oriented lines of $\mathbb{R}^{7}=\operatorname{Im} \mathbb{O}$ which is invariant by the transitive action of $G_{2} \ltimes \mathbb{R}^{7}$, where $G_{2}$ is the automorphism group of the octonions $\mathbb{O}$.

3. Null, space- and time-like curves. In order to give a geometric interpretation for a curve in $\mathcal{G}$ endowed with some of the $G$-invariant metrics to be null, space- or time-like, we introduce the following concept, which makes sense for any Hadamard manifold.

Definition. Let $H$ be a Hadamard manifold. Given a smooth curve $c$ in $\mathcal{G}$ defined on the interval $I$, a function $\varphi: \mathbb{R} \times I \rightarrow H$ is said to be a standard presentation of $c$ if $s \mapsto \alpha_{t}(s):=\varphi(s, t)$ is a unit speed geodesic of $H$ satisfying $c(t)=\left[\alpha_{t}\right]$ and $\left\langle\dot{\beta}(t), \dot{\alpha}_{t}(0)\right\rangle=0$ for all $t \in I$, where $\beta(t)=\varphi(0, t)$.

Proposition 3. Given a smooth curve $c: I \rightarrow \mathcal{G}$ and $p$ a point in the image of some (any) geodesic in the equivalence class $c\left(t_{o}\right)$, there exists a standard presentation $\varphi$ of $c$ such that $\varphi\left(0, t_{o}\right)=p$.

Proof. Consider the submersion $\Pi: T^{1} H \rightarrow \mathcal{G}, \Pi(v)=\left[\gamma_{v}\right]$. Let $v(t)$ be a lift of $c(t)$ to $T^{1} H$ with $v\left(t_{o}\right) \in T_{p}^{1} H$, and let $\psi: \mathbb{R} \times I \rightarrow H$ be defined by $\psi(s, t)=\gamma_{v(t)}(s)$. 
We look for a function $f: I \rightarrow \mathbb{R}$ such that

$$
\varphi(s, t)=\psi(s+f(t), t)
$$

satisfies the required properties. Clearly $\alpha_{t}(s)=\varphi(s, t)$ has unit speed and

$$
c(t)=\Pi(v(t))=\Pi\left(\gamma_{v(t)}^{\prime}(f(t))\right)=\left[\alpha_{t}\right] .
$$

One can verify easily that taking as $f$ the solution of the differential equation

$$
f^{\prime}(t)=-\frac{\left\langle\psi_{t}(f(t), t), \psi_{s}(f(t), t)\right\rangle}{\left\|\psi_{s}(f(t), t)\right\|^{2}}
$$

(subscripts denote partial derivatives) with $f\left(t_{o}\right)=0$, then $\varphi\left(0, t_{o}\right)=p$ and $\left\langle\dot{\beta}(t), \dot{\alpha}_{t}(0)\right\rangle=0$ for all $t \in I$, where $\beta$ is as in the definition of the standard presentation.

The following proposition characterizes the null, time- and space-like curves of $\mathcal{G}$, providing a relationship between the geometries of $\mathcal{G}$ and $H$.

Proposition 4. For the metric $g_{1}$, a smooth curve $c$ in $\mathcal{G}_{n}$ is null (respectively, space-, time-like) if and only if, for any standard presentation, the rate of variation of the directions, that is, $\left\|\frac{D}{d t} \dot{\alpha}_{t}(0)\right\|$, coincides with (respectively, is smaller, larger than) the rate of displacement $\|\dot{\beta}(t)\|$ for all $t$ (here $\frac{D}{d t}$ denotes covariant derivative along $\beta$ ).

For the metric $g_{0}$ on $_{\mathcal{G}_{3}}$, a smooth curve $c$ in $\mathcal{G}_{3}$ is null (respectively, space-, time-like) if and only if, for any standard presentation,

$$
\left\{\dot{\beta}(t), \frac{D}{d t} \dot{\alpha}_{t}(0), \dot{\alpha}_{t}(0)\right\}
$$

is linearly dependent (respectively, positively, negatively oriented) for all $t$.

Proof. Let $[\gamma]$ be an oriented geodesic of a Hadamard manifold and let $\mathcal{J}_{\gamma}$ be the space of Jacobi fields along $\gamma$ orthogonal to $\dot{\gamma}$. First we show that $L_{\gamma}: \mathcal{J}_{\gamma} \rightarrow T_{[\gamma]} \mathcal{G}$ given by

$$
L_{\gamma}(J)=(d / d t)_{0}\left[\gamma_{t}\right]
$$

where $\gamma_{t}$ is a variation of $\gamma$ by unit speed geodesics associated with the Jacobi field $J$, is a well-defined vector space isomorphism. Indeed, let $\Pi: T^{1} M \rightarrow \mathcal{G}$ be as above the canonical projection, which is a smooth submersion, by definition of the differentiable structure on $\mathcal{G}$. We compute

$$
(d / d t)_{0}\left[\gamma_{t}\right]=(d / d t)_{0} \Pi\left(\dot{\gamma}_{t}(0)\right)=d \Pi_{\dot{\gamma}(0)}\left((d / d t)_{0} \dot{\gamma}_{t}(0)\right) .
$$

Now, let $\mathrm{p}: T^{1} H \rightarrow H$ be the canonical projection and $\mathcal{K}: T_{\dot{\gamma}(0)}\left(T^{1} H\right) \rightarrow \dot{\gamma}(0)^{\perp} \subset$ $T_{\gamma(0)} H$ the connection operator. It is well-known that $(d \mathrm{p}, \mathcal{K}): T_{\dot{\gamma}(0)}\left(T^{1} H\right) \rightarrow$ $T_{\gamma(0)} H \oplus \dot{\gamma}(0)^{\perp}$ is a bijection and

$$
(d / d t)_{0} \dot{\gamma}_{t}(0)=(d \mathrm{p}, \mathcal{K})^{-1}\left(J(0), J^{\prime}(0)\right)
$$

(see for instance [1]). Therefore, $L_{\gamma}$ is well-defined. 
Next we show that for any $J \in \mathcal{J}_{\gamma}$ one has

$$
\begin{aligned}
\left\|L_{\gamma}(J)\right\|_{1} & =\|J(0)\|^{2}-\left\|J^{\prime}(0)\right\|^{2}, \\
\left\|L_{\gamma}(J)\right\|_{0} & =\left\langle\dot{\gamma}(0) \times J(0), J^{\prime}(0)\right\rangle .
\end{aligned}
$$

We may suppose without loss of generality that $c=c_{o}$ and $\gamma=\gamma_{o}$. Let $c^{\prime}(0)=x_{h}+y_{v}$ with $x, y \in \mathbb{R}^{n}$. Then the Jacobi field along $\gamma_{o}$ satisfying $L_{\gamma_{o}}(J)=c^{\prime}(0)$ is the one determined by

$$
J(0)=d \pi_{I}\left(x_{h}\right) \text { and } J^{\prime}(0)=d \pi_{I}\left(y_{h}\right)
$$

where $\pi: G \rightarrow H$ is as before the canonical projection. In fact, clearly, $\gamma_{t}(s)=$ $\exp \left(t x_{h}\right) \exp \left(t y_{v}\right) \gamma_{o}(s)$ is a variation of $\gamma_{o}$ by unit speed geodesics. Let us see that the associated Jacobi field is $J$. Indeed,

$$
J(0)=\left.\frac{d}{d t}\right|_{0} \gamma_{t}(0)=\left.\frac{d}{d t}\right|_{0} \exp \left(t x_{h}\right) e_{0}=d \pi_{I}\left(x_{h}\right)
$$

since $\gamma_{o}(0)=e_{0}$, which is fixed by $\exp \left(t y_{v}\right)$. If $\frac{D}{d t}$ denotes covariant derivative along $t \mapsto \gamma_{t}(0)$ and $Z$ is as in the beginning of the proof of Theorem 1 , then

$$
\begin{aligned}
J^{\prime}(0) & =\left.\frac{D}{d t}\right|_{0} \dot{\gamma}_{t}(0)=\left.\frac{D}{d t}\right|_{0} d\left(\exp \left(t x_{h}\right) \exp \left(t y_{v}\right)\right)_{\pi(I)} e_{1} \\
& =\left.\frac{D}{d t}\right|_{0} d \exp \left(t x_{h}\right) d \pi_{I} \operatorname{Ad}\left(\exp t y_{v}\right) Z \\
& =\left.d \pi_{I} \frac{d}{d t}\right|_{0} e^{t \text { ad } y_{v}} Z=d \pi_{I}\left[y_{v}, Z\right]=d \pi_{I}\left(y_{h}\right),
\end{aligned}
$$

since $d \exp \left(t x_{h}\right)$ realizes the parallel transport and $d \pi_{I}(Z)=e_{1}$. Therefore (2) is true by Theorem 1. Finally, suppose that $\varphi$ is a standard presentation of $c$ and let $\alpha_{t}, \beta$ be as above. Let $J_{t}$ denote the Jacobi field along $\alpha_{t}$ associated with the variation $\varphi$. Clearly, $\dot{c}(t)=L_{\alpha_{t}}\left(J_{t}\right), J_{t}(0)=\frac{d}{d t} \varphi(0, t)=\dot{\beta}(t)$ and

$$
J_{t}^{\prime}(0)=\left.\frac{D}{d s}\right|_{0} \frac{d}{d t} \varphi(s, t)=\left.\frac{D}{d t} \frac{d}{d s}\right|_{0} \varphi(s, t)=\frac{D}{d t} \dot{\alpha}_{t}(0) .
$$

Consequently, the proposition follows from (2).

4. A geometric invariant of $\mathcal{G}$. We have mentioned in the introduction that $\mathcal{G}\left(H^{n}\right)$ is diffeomorphic to $\mathbb{T}^{n}$, the space of all oriented lines of $\mathbb{R}^{n}$. For $n=3$ and $n=7$, we found in [9] pseudo-Riemannian metrics on $\mathbb{T}^{n}$ invariant by the induced transitive action of a connected closed subgroup of $S O_{n} \ltimes \mathbb{R}^{n}$ (only for those dimensions such metrics exist).

PROPOSITION 5. For $n=3,7$, no metric on $\mathcal{G}_{n}$ invariant by the identity component of the isometry group of $H^{n}$ is isometric to $\mathbb{T}^{n}$ endowed with any of the metrics above.

Proof. We compute now a pseudo-Riemannian invariant of $\mathcal{G}_{n}$ involving its periodic geodesics. For any $c \in \mathcal{G}$, let $A$ denote the subset of $T_{c} \mathcal{G}$ consisting of the velocities of periodic geodesics of $\mathcal{G}$ though $c$. We show next that the frontier of $A$ in $T_{c} \mathcal{G}$ is the union of two subspaces of half the dimension of $\mathcal{G}$ intersecting only at zero. 
By homogeneity we may suppose that $c=c_{o}$. Since by the proposition below $A=$ $\left\{\lambda x_{h}+x_{v}\left|x \in \mathbb{R}^{n},\right| \lambda \mid<1\right\}$, the frontier of $A$ is $\mathfrak{g}_{1} \cup \mathfrak{g}_{-1}$. On the other hand, we have computed in [10] that the analogue invariant for $\mathbb{T}^{n}(n=3,7)$ is a subspace of half the dimension of $\mathbb{T}^{n}$. Hence the proposition follows.

REMARKS. a) Of course we could have considered more standard invariants, like the curvature or the isometry group, but we chose this one since the geodesics can be described so easily.

b) Clearly the difference in the invariants is related to the fact that the two horospheres through a point associated with opposite directions coincide in the Euclidean case but are different in the hyperbolic case.

Proposition 6. A geodesic in $\mathcal{G}$ with initial velocity $x_{h}+y_{v}$ is periodic if and only if $x=\lambda y$ for some $\lambda \in \mathbb{R}$ with $|\lambda|<1$.

Proof. We may suppose that $x_{h}+y_{v} \neq 0$. We compute that $\operatorname{Ad}\left(e^{t Z}\right)\left(x_{h}+y_{v}\right)=$ $x_{v}^{t}+y_{v}^{t}$, where

$$
x^{t}=(\cosh t) x+(\sinh t) y \quad \text { and } \quad y^{t}=(\sinh t) x+(\cosh t) y .
$$

Now, there exists $s$ such that $\left\langle x^{s}, y^{s}\right\rangle=0\left(\operatorname{take} \tanh (2 s)=-\frac{2\langle x, y\rangle}{|x|^{2}+|y|^{2}}\right)$. Hence $\left[x_{h}^{s}, y_{v}^{s}\right]=0$ and consequently

$$
\pi \exp \left(t\left(x_{h}^{s}+y_{v}^{s}\right)\right)=\pi \exp \left(t x_{h}^{s}\right) \exp \left(t y_{v}^{s}\right)=\pi \exp \left(t x_{h}^{s}\right)
$$

which is a geodesic in $H$, in particular it is periodic only if it is constant, or equivalently, only for $x^{s}=0$.

Since $Z \in \mathfrak{g}_{0}$ and the metric is $G$-invariant, the geodesics with initial velocities $x_{h}^{t}+y_{v}^{t}$ are simultaneously periodical or not periodical for all $t$. Now, one verifies that $x^{s}=0$ if and only if $x=\lambda y$ for some $\lambda \in \mathbb{R}$ with $|\lambda|<1$ and the proposition follows.

5. Additional geometric structures on $\mathcal{G}$. An almost Hermitian structure on a pseudo-Riemannian manifold $(M, g)$ is a smooth tensor field $J$ of type $(1,1)$ on $M$ such that $J_{p}$ is an orthogonal transformation of $\left(T_{p} M, g_{p}\right)$ and satisfies $J_{p}^{2}=-\mathrm{id}$ for all $p \in M$. If $\nabla$ is the Levi Civita connection of $(M, g)$, then $(M, g, J)$ is said to be Kähler if $\nabla J=0$.

A Kähler structure on $\mathcal{G}\left(H^{3}\right)$. Let $\mathcal{G}=\mathcal{G}_{3}$ and let $j_{o}$ be the endomorphism of $\mathfrak{h} \equiv$ $T_{c_{o}} \mathcal{G} \equiv \mathbb{C} \times \mathbb{C}$ given by $j_{o}(z, w)=(i z, i w)$. One checks that $j_{o}$ commutes with the action of $G_{o}$, is orthogonal for $g_{0}$ and $g_{1}$ and $j_{o}^{2}=-\mathrm{id}$. Therefore $j_{o}$ defines an orthogonal almost complex structure on $\mathcal{G}_{3}$ for any $G$-invariant metric on it.

Proposition 7. The space $\left(\mathcal{G}_{3}, J\right)$ is Kähler for any pseudo-Riemannian G-invariant metric on $\mathcal{G}_{3}$.

Proof. We show that for every geodesic $\gamma$ in $\mathcal{G}_{3}$ and any parallel vector field $Y$ along $\gamma$, the vector field $J Y$ along $\gamma$ is parallel. By homogeneity we may suppose that $\gamma(0)=c_{o}$. Suppose that $\gamma(t)=\exp (t X) c_{o}$ for some $X \in \mathfrak{h}$. By a well-known property of 
symmetric spaces, $Y=d \exp (t X)_{c_{o}} Y_{c_{o}}$. Since $J$ is $G$-invariant, $J Y=d \exp (t X)_{c_{o}} J Y_{c_{o}}$ and thus $J Y$ is parallel along $\gamma$, as desired.

An orthogonal almost complex structure on $\mathcal{G}_{7}$. We present another model of $\mathcal{G}_{n+1}$ endowed with the metric $g_{1}$ and use it to define an orthogonal almost complex structure on $\mathcal{G}_{7}$.

In the following we use the notations given before Proposition 2 of concepts related to the imaginary border of $H$. We recall that $g \in G$ is called a transvection of $H$ if it preserves a geodesic $\gamma$ of $H$ and $d g$ realizes the parallel transport along $\gamma$, that is, $g(\gamma(t))=\gamma(t+s)$ for all $t$ and some $s$ and $d g_{\gamma(t)}$ realizes the parallel transport between $\gamma(t)$ and $\gamma(t+s)$ along $\gamma$. For any unit $v \in T_{e_{0}} H=e_{0}^{\perp}=\mathbb{R}^{n+1}$ the transvections through $e_{0} \in H$ preserving the geodesic with initial velocity $v$ form a one parameter subgroup $\phi_{t}$ such that the corresponding one parameter group $\widetilde{\phi}_{t}$ of conformal transformations of $S^{n}$ (which we also call transvections, by abuse of notation) is the flow of the vector field on $S^{n}$ defined at $q \in S^{n}$ as the orthogonal projection of the constant vector field $v$ on $\mathbb{R}^{n+1}$ onto $T_{q} S^{n}=q^{\perp}$. In particular $\widetilde{\phi}_{t}$ fixes $\pm v \in S^{n}$. For $\tau=\widetilde{\phi}_{t}$ we will need specifically the following standard facts:

*) If $u \in S^{n}$ is orthogonal to $v$, then $v \in T_{u} S^{n}$ and if $\tau(u)=(\cos \theta) u+(\sin \theta) v$, then $(d \tau)_{u} v$ is a vector in $T_{\tau(u)} S^{n}$ spanned by $u$ and $v$ of length $\cos \theta$.

**) There exists a positive constant $c$ such that $(d \tau)_{ \pm v}$ is a multiple $c^{ \pm 1}$ of the identity map on $T_{ \pm v} S^{n}=v^{\perp}$.

Let $\Delta_{n}=\left\{(p, p) \mid p \in S^{n}\right\}$ denote the diagonal in $S^{n} \times S^{n}$. The map

$$
\psi: \mathcal{G}_{n+1} \rightarrow\left(S^{n} \times S^{n}\right) \backslash \Delta_{n}, \quad \psi([\gamma])=(\gamma(-\infty), \gamma(\infty))
$$

is a well-defined diffeomorphism. We denote by $\widehat{g}$ the induced action of $g \in G$ on $\left(S^{n} \times S^{n}\right) \backslash \Delta_{n}$, that is $\widehat{g}(p, q)=(\widetilde{g}(p), \widetilde{g}(q))$. Given distinct points $p, q \in S^{n}$, let $T_{p, q}$ denote the reflection on $\mathbb{R}^{n+1}$ with respect to the hyperplane orthogonal to $p-q$.

Proposition 8. If $\mathcal{G}_{n+1}$ is endowed with the metric $g_{1}$ and one considers on $\left(S^{n} \times\right.$ $\left.S^{n}\right) \backslash \Delta_{n}$ the pseudo-Riemannian metric whose associated norm is

$$
\|(x, y)\|_{(p, q)}=4\left\langle T_{p, q} x, y\right\rangle /|q-p|^{2}
$$

for $x \in p^{\perp}, y \in q^{\perp}$, then the diffeomorphism $\psi$ of (3) is an isometry.

Proof. Clearly $\psi$ is $G$-equivariant. Since the metric $g_{1}$ on $\mathcal{G}_{n+1}$ is $G$-invariant, it is sufficient to show that the metric (4) on $\left(S^{n} \times S^{n}\right) \backslash \Delta_{n}$ is $G$-invariant as well and that $d \psi_{\left[\gamma_{0}\right]}$ is a linear isometry.

Given distinct points $p_{ \pm} \in S^{n}$, we show first that for any $g \in G$ with $\widetilde{g}\left(e_{ \pm 1}\right)=p_{ \pm}$, $d \widehat{g}_{\left(-e_{1}, e_{1}\right)}$ is a linear isometry. A straightforward computation shows that the given metric on $\left(S^{n} \times S^{n}\right) \backslash \Delta_{n}$ is invariant by the action of $S O_{n+1}$, since for all $k$ in this group, $T_{k(p), k(q)} \circ k=k \circ T_{p, q}$ for all $p, q \in S^{n}, p \neq q$. Hence we may suppose without loss of generality that $p_{ \pm}= \pm(\cos \theta) e_{1}+(\sin \theta) e_{2}$ for some $\theta \in[0, \pi / 2)$. Now, any directly conformal transformation $\widetilde{g}$ as above may be written as a composition $\tau^{2} \circ \tau^{1} \circ R$, where $R$ is a rotation fixing $e_{1}$ and $\tau^{1}$ and $\tau^{2}$ are transvections fixing $\left(-e_{1}, e_{1}\right)$ and $\left(-e_{2}, e_{2}\right)$, respectively.

The assertion $(* *)$ above, with $v=e_{1}$ and $\tau=\tau^{1}$, implies that $d \widehat{\tau}_{\left(-e_{1}, e_{1}\right)}$ is a linear isometry. Now we use the assertion $(*)$ with $v=e_{2}$ and $u=e_{1}$ to see that 
$d \widehat{\tau^{2}}{ }_{\left(-e_{1}, e_{1}\right)}: e_{1}^{\perp} \times e_{1}^{\perp} \rightarrow p_{-}^{\perp} \times p_{+}^{\perp}$ is a linear isometry. Let $\lambda_{ \pm} v+x_{ \pm} \in T_{ \pm u} S^{n}=u^{\perp}$, with $\lambda_{ \pm}$real numbers and $\left\langle x_{ \pm}, v\right\rangle=0$. One computes

$$
\begin{aligned}
\left\|\left(\lambda_{-} v+x_{-}, \lambda_{+} v+x_{+}\right)\right\|_{(-u, u)} & =4\left(\lambda_{-} \lambda_{+}+\left\langle x_{-}, x_{+}\right\rangle\right)|2 u|^{2} \\
& =\left(\lambda_{-} \lambda_{+}+\left\langle x_{-}, x_{+}\right\rangle\right) .
\end{aligned}
$$

On the other hand, call $d \tau_{ \pm u}^{2}(v)=v_{ \pm}$and $d \tau_{ \pm u}^{2}\left(x_{ \pm}\right)=y_{ \pm}$. Hence $\left|v_{ \pm}\right|=\cos \theta$. Since $d \tau_{ \pm u}^{2}$ is conformal, $y_{ \pm}$is orthogonal to $v_{ \pm}$and has length $\left|x_{ \pm}\right| \cos \theta$. Also, $y_{ \pm}$is orthogonal to $u$, hence it is left fixed by $T_{p_{-}, p_{+}}$. Therefore one computes

$$
\left\|\left(\lambda_{-} v_{-}+y_{-}, \lambda_{+} v_{+}+y_{+}\right)\right\|_{(-u, u)}=\frac{4 \cos ^{2} \theta}{\left|p_{-}-p_{+}\right|^{2}}\left(\lambda_{-} \lambda_{+}+\left\langle x_{-}, x_{+}\right\rangle\right),
$$

which coincides with (5) since $\left|p_{-}-p_{+}\right|=2 \cos \theta$. This completes the proof that $d \widehat{g}_{\left(-e_{1}, e_{1}\right)}$ is a linear isometry. It remains only to show that $d \psi_{\left[\gamma_{o}\right]}$ is a linear isometry.

We have that $\gamma_{o}(t)=(\cosh t, \sinh t, 0) \in \mathbb{R}^{n+2}$. Let $J$ be the Jacobi field along $\gamma_{o}$ orthogonal to $\gamma_{o}$ and satisfying $J(0)=x$ and $J^{\prime}(0)=y$, both in $T_{e_{0}} H$ orthogonal to $e_{1}=\gamma^{\prime}(0)$. We show next that

$$
d \psi_{\left[\gamma_{o}\right]} L_{\gamma_{o}}(J)=(x-y, x+y),
$$

where $L_{\gamma_{o}}$ was defined in (1). By invariance of $\psi$ by rotations it is sufficient to see that

$$
d \psi_{\left[\gamma_{o}\right]} L_{\gamma_{o}}\left(J_{ \pm}\right)=\left( \pm e_{2}, e_{2}\right),
$$

where $J_{-}(0)=0, J_{-}^{\prime}(0)=e_{2}, J_{+}(0)=e_{2}$ and $J_{+}^{\prime}(0)=0$. Let now

$$
A_{s}=\left(\begin{array}{cc}
\cos s & -\sin s \\
\sin s & \cos s
\end{array}\right) \text { and } B_{s}=\left(\begin{array}{ccc}
\cosh s & 0 & \sinh s \\
0 & 1 & 0 \\
\sinh s & 0 & \cosh s
\end{array}\right) \text {. }
$$

The field $J_{-}$is associated to the variation of $\gamma_{o}$ corresponding to the one parameter group of isometries $s \mapsto A_{s}^{-}=\operatorname{diag}\left(1, A_{s}, I_{n-1}\right)$. One computes $A_{s}^{-}\left(\gamma_{o}(t)\right)=$ $(\cosh t) e_{0}+\sinh t\left((\cos s) e_{1}+(\sin s) e_{2}\right) \in H$. Hence

$$
\begin{aligned}
\left(A_{s}^{-} \circ \gamma_{o}\right)( \pm \infty) & =\lim _{t \rightarrow \pm \infty}(\tanh t)\left((\cos s) e_{1}+(\sin s) e_{2}\right) \\
& = \pm(\cos s) e_{1} \pm(\sin s) e_{2},
\end{aligned}
$$

whose derivative at $s=0$ is $\pm e_{2}$. Therefore $\left(d /\left.d s\right|_{0}\right) \psi\left[A_{s}^{-} \circ \gamma_{o}\right]=\left(-e_{2}, e_{2}\right)$. Using $B_{s}^{+}=$ $\operatorname{diag}\left(B_{s}, I_{n-1}\right)$ instead of $A_{s}^{-}$one verifies the remaining identity of (6). Finally, since $T_{-e_{1}, e_{1}}$ clearly fixes $x, y$, the norm (4) of $(x-y, x+y)$ at $\left(-e_{1}, e_{1}\right)$ is $4\langle x-y, x+$ $y\rangle /\left|2 e_{1}\right|^{2}=|x|^{2}-|y|^{2}$, which coincides with the norm of $L_{\gamma_{o}}(J)$ by (2). This shows that $d \psi_{\left[\gamma_{o}\right]}$ is a linear isometry.

Let $\mathbb{O}$ denote the normed division algebra of the octonions and let $\mathbb{R}^{7}=\operatorname{Im} \mathbb{O}$ endowed with its canonical cross product $\times$. Let $j$ be the almost complex structure of $S^{6}$ defined by $j_{p}(x)=p \times x$ if $x \in T_{p} S^{6}=p^{\perp}$. For $q \in S^{6}, q \neq p$, let $j_{p, q}$ be the linear operator on $T_{q} S^{6}=q^{\perp}$ defined by $j_{p, q}=T_{p, q} \circ j_{p} \circ T_{p, q}$. 
Proposition 9. For all $x \in p^{\perp}, y \in q^{\perp}$,

$$
J_{(p, q)}(x, y)=\left(j_{p}(x), j_{p, q}(y)\right)
$$

defines an orthogonal almost complex structure on $\left(S^{6} \times S^{6}\right) \backslash \Delta_{n}$ with the metric above.

Proof. First we check that $J$ is an almost complex structure. Indeed,

$$
\left\langle j_{p, q}(y), q\right\rangle=\left\langle j_{p} T_{p, q}(y), T_{p, q}(q)\right\rangle=\left\langle p \times T_{p, q}(y), p\right\rangle=0
$$

and $J^{2}=-$ id holds as well, since $j_{p}^{2}=-$ id and $T_{p, q}^{2}=$ id. Finally, $J$ is orthogonal since both $j_{p}$ and $T_{p, q}$ are so.

REMARKS. a) By Proposition 2 there exists no proper subgroup of $G$ acting transitively on $\mathcal{G}$ leaving $J$ invariant, as it is the case of the analogous almost complex structure defined in [9] on the space of oriented lines of $\mathbb{R}^{7}$.

b) The structure $J$ is not integrable, since $\left(S^{6} \backslash\{p\}\right) \times\{p\}$ is an almost complex submanifold for any $p$, whose induced almost complex structure is $q \mapsto j_{q}$, which is not integrable.

ACKNOWLEDGEMENTS. I would like to thank Eduardo Hulett for his help concerning the symmetry of $\mathcal{G}$ and Antonio Di Scala for the statement and the idea of the proof of Proposition 2.

\section{REFERENCES}

1. A. Besse, Manifolds all of whose geodesics are closed, Ergebnisse der Mathematik und ihre Grenzgebiete no. 93 (Springer-Verlag, 1978).

2. A. Di Scala and C. Olmos, The geometry of homogeneous submanifolds of hyperbolic space, Math. Z. 237 (2001), 199-209.

3. B. Guilfoyle and W. Klingenberg, On the space of oriented affine lines in $\mathbb{R}^{3}$, Archiv Math: (Basel). 82 (2004), 81-84.

4. B. Guilfoyle and W. Klingenberg, An indefinite Kähler metric on the space of oriented lines, J. London Math. Soc. (2). 72 (2005), 497-509.

5. N. J. Hitchin, Monopoles and geodesics, Comm. Math. Phys. 83 (1982), 579-602.

6. M. Kanai, Geodesic flows of negatively curved manifolds with smooth stable and unstable foliations, Ergodic Theory Dyn. Syst. 8 (1988), 215-239.

7. G. Keilhauer, A note on the space of geodesics, Rev. Unión Mat. Argent. 36 (1990), $164-173$

8. R. Palais, A global formulation of the Lie theory of transformation groups, Memoirs American Math. Soc. No. 22 (1957)

9. M. Salvai, On the geometry of the space of oriented lines in Euclidean space, Manuscr. Math. 118 (2005), 181-189.

10. M. Salvai, Geodesics in the space of oriented lines in Euclidean space, Proceedings of Egeo 2005, Rev. Unión Mat. Argent. (2) 47 (2006), 109-114.

11. M. Shepherd, Line congruences as surfaces in the space of lines. Diff. Geom. Appl. 10 (1999), 1-26. 